\title{
The intervals at which homogeneous flashes recover masked targets
}

\author{
E. TENKINK and J. H. WERNER \\ University of Leiden, 2312 KM Leiden, The Netherlands
}

\begin{abstract}
The recovery effect may be induced by the introduction of a homogeneous blank flash after a patterned mask. It is not clear which parameters optimize the effect. In this study, the effect of systematical variation of the different interstimulus intervals on performance was investigated. Conditions in which the blank flash preceded the patterned mask were included. The important parameter is shown to be the interval between Mask 1 and Mask 2. For optimal recovery, this value has to be $0-20$ msec. It is argued that these results, as well as a number of other recovery findings, can be explained by the suppressing influence of homogeneous flashes on contour processing channels in the visual system.
\end{abstract}

The presentation of a pattern (M) or blank (B) flash shortly before or after a target presentation (T) generally has a negative effect on the identification performance. On the other hand, it may be possible to improve detectability of a masked target $(T+M)$ by the presentation of a third, subsequent flash (recovery). Such recovery was first reported by Robinson (1966). In further experiments, he was able to reproduce and extend his findings (Robinson, 1968). His results are thought to have implications for the generality of integration theories of visual masking (Dember, Schwartz, \& Kocak, 1978).

The interpretation of the results of Robinson (1966, 1968 ) is rather difficult due to a number of peculiarities in his design. As Schurman and Eriksen (1969) have pointed out, a number of cues (including brightness differences and apparent movement) might have facilitated the detection of the target flash. These investigators made substantial improvements on the experimental design and tried to reproduce Robinson's results. However, they found no recovery of the masked target. A letter identification experiment likewise yielded negative results.

At the same time, a number of positive results concerning recovery have been reported. The results presented by Dember and Purcell (1967) showed moderate recovery. Recently, Kristofferson, Galloway, and Hanson (1979) repeated the experiment and found an almost complete recovery of the masked target. Dember, Schwartz, and Kocak (1978) and Schiller and Greenfield (1969) have also reported positive instances of recovery using different stimulus configurations.

It is not clear which parameters optimize the effect. Absolute and relative stimulus size, the intensity and

Reprints may be obtained from E. Tenkink, Department of Psychology, University of Leiden, Hooigracht 15, 2312 KM Leiden, The Netherlands. duration of the flashes, and the temporal intervals between the different stimuli have not yet been studied extensively. Systematic variation of these intervals is necessary to elucidate the temporal organization of the mechanisms that are responsible for the effect. This approach may give detailed information about the localization of the recovery optima in relative temporal coordinates. In addition, the temporal extent of the effect may become clear. Kristofferson et al. (1979) introduced a slight variation only in the time intervals and concluded that the optimal interval between the masks for the induction of recovery ( $35 \mathrm{msec}$ ) was that originally chosen by Dember and Purcell (1967).

Both Robinson (1968) and Schiller and Greenfield (1969) addressed this question in greater detail. However, their results do not allow a very precise estimation of the optimum or the temporal extent of the effect.

In the present study, we varied independently the interval between $T$ and $M\left(t_{1}\right)$ and the interval between $T$ and $B\left(t_{2}\right)$ over a large range. We deliberately introduced a number of conditions in which $B$ preceded M. Furthermore, we took care to avoid a number of possible confounding factors in the experiments (Schurman \& Eriksen, 1969).

\section{METHOD}

\section{Subjects}

Four adult males (24-32 years) served as subjects. Three of them were students, the fourth was one of the authors. All subjects had normal or corrected-to-normal vision.

\section{Apparatus}

The stimuli were generated on a fast display screen (VectorGeneral) equipped with $P_{3}$ phosphor. Stimulus presentation and response registration were controlled by a PDP-11/04 minicomputer. The frame-length was $10 \mathrm{msec}$. A square area $(28 \times$ $28 \mathrm{deg}$ ) centered round the fixation dot served as a black background field. All patterns were presented white on black. Outside 
this area, the room was dimly illuminated $\left(1.5 \mathrm{cdm}^{-2}\right)$. The numbers 1 to 9 were used as targets.

Subjects had to identify the target $(T)$ by pressing the corresponding key on a 3 by 3 response panel. At the viewing distance of $160 \mathrm{~cm}$, line width was $1 \mathrm{~min}$. Target height was $.28 \mathrm{deg}$, and target width was $.20 \mathrm{deg}$. The noise mask (M) consisted of a $\overline{7}$ by 7 dot matrix (dot diameter, $1 \mathrm{~min}$ ). Each dot had a .5 probability of appearing. The average number of dots present was 25 . The mask dimensions were $.4 \times .4 \mathrm{deg}$. The blank flash (B) extended $.4 \times .4 \mathrm{deg}$.

The luminances for target $\left(.19 \mathrm{cdm}^{-2}\right)$, noise $\left(18 \mathrm{cdm}^{-2}\right)$, and blank flash $\left(80 \mathrm{cdm}^{-2}\right)$ were measured across the $.4 \times .4 \mathrm{deg}$ field. These luminances were determined in pilot studies and yielded approximately equal masking effects for the noise flash and the blank flash. The target presented alone was perfectly discriminable. Viewing was binocular, and all stimuli were presented foveally.

Both target and noise-mask duration were $20 \mathrm{msec}$. The blank flash was presented for $10 \mathrm{msec}$. The intervals between target onset and mask onset $\left(t_{1}\right)$ ranged from 40 to $100 \mathrm{msec}$ in 20 -msec units. The intervals between target onset and blank-field onset $\left(\mathrm{t}_{2}\right)$ were varied likewise, from 40 to $220 \mathrm{msec}$. Masking curves for $T+M$ and $T+B$ alone were obtained in the range of 40 100 msec at 10 -msec intervals.

\section{Procedure}

The subjects received $1 \mathrm{~h}$ of training to acquaint themselves with the stimulus conditions and the response method. Each experimental session was started with a number of warming-up blocks. This period also served as a dark-adaptation period $(20 \mathrm{~min})$.

Within each block ( 27 trials), each of the available numbers was presented three times. In each block, only one combination of $t_{1}$ and $t_{2}$ was used. The order of presentation of the combinations (including $t_{1}$ and $t_{2}$ alone) was random. The total experiment was run in two 3-h sessions.

Observers initiated a trial by pressing a request key. Slight dimming of the fixation dot served as a warning period $(500 \mathrm{msec})$ before the start of the stimulus sequence. The stimuli replaced the fixation dot. After the subject's response, the sequence was repeated.

\section{RESULTS}

The proportion of correct responses was taken as a measure of performance. This measure was not corrected for chance. Since the results for all subjects were essentially similar, we pooled the data. Figure 1 summarizes the results of our experiments. The simple noise-masking curve is presented in Figure 1A. Increase of performance occurs between $t_{1}=40 \mathrm{msec}$ and $t_{1}=$ $100 \mathrm{msec}$. The simple blank-flash masking curve is shown in Figure 1B. In this case, detection performance increases monotonically from chance level at $t_{2}=40 \mathrm{msec}$ to almost perfect performance at $70 \mathrm{msec}$.

The detection performance in the condition in which target, mask, and blank flash are presented is shown in Figure $1 C$ for different values of $\Delta t=t_{2}-t_{1}$. The recovery effect is defined as a positive difference in detection performance between the condition $\mathrm{T}+\mathrm{M}+\mathrm{B}$ and the condition $\mathrm{T}+\mathrm{M}$.

To test the effect of $t_{2}$ on these differences, we performed four separate analyses of variance. For each $t_{1}$ level, the effect of $t_{2}$ was significant: smallest $F(9,27)$ $=3.3, p<.05$. Subsequently, the average difference scores were compared by means of Newman-Keuls tests (significance level .05). At $t_{1}=40 \mathrm{msec}$, the per-
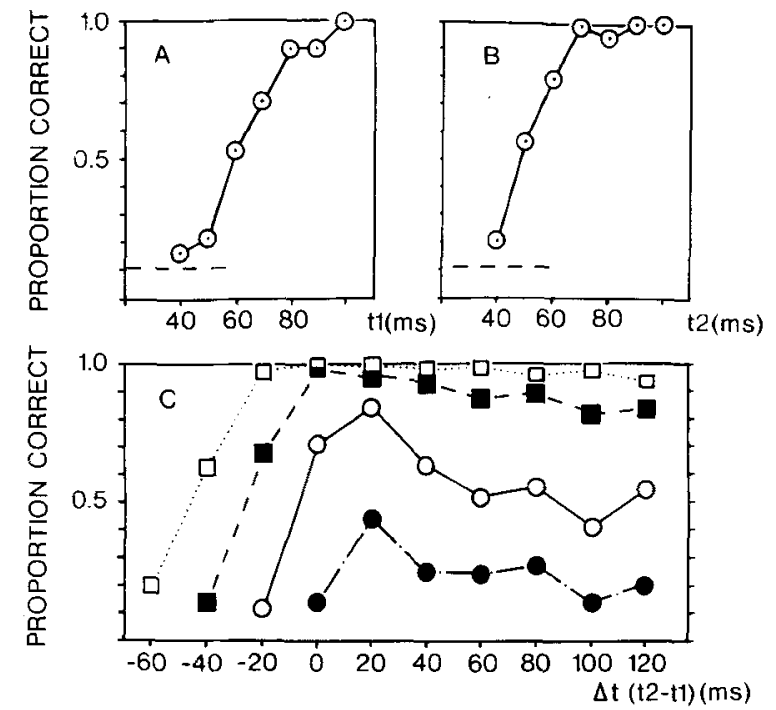

Figure 1. (A and B) The effects on performance of one masking flash (noise flash and blank nash, respectively) as a function of the interval between target onset and mask onset. (C) The effects of two consecutive masking flashes at different $t_{1}$ values: $\bullet t_{1}=$ $40 \mathrm{msec}, O t_{1}=60 \mathrm{msec}, \square t_{1}=80 \mathrm{msec}, \square t_{1}=100 \mathrm{msec}$.

formance at $\Delta t=20 \mathrm{msec}$ was significantly better than it was at other $\Delta t$ values. A similar result was obtained at $\mathrm{t}_{1}=60 \mathrm{msec}$. The performance at $\Delta \mathrm{t}=20 \mathrm{msec}$ was significantly better than at other $\Delta t$ values except at $\Delta \mathrm{t}=0$ msec.

A statistically reliable positive difference (i.e., recovery) was found at $\Delta t=20 \mathrm{msec}$ for $t_{1}=40 \mathrm{msec}$ and at $\Delta t=20 \mathrm{msec}$ and $0 \mathrm{msec}$ for $\mathrm{t}_{1}=60 \mathrm{msec}$. No significant recovery effects could be demonstrated at $t_{1}=80 \mathrm{msec}$ and $t_{1}=100$ msec. However, as can be seen from Figure 1C, maximum performance in these cases is found at $\Delta t=0 \mathrm{msec}$. This location is in agreement with the results obtained at $t_{1}=40 \mathrm{msec}$ and $t_{1}=60 \mathrm{msec}$. We attribute the absence of statistical significance at $t_{1}=80 \mathrm{msec}$ and $t_{1}=100 \mathrm{msec}$ to ceiling effects in performance.

At negative $\Delta t$ values, the performance is significantly worse than it is at other $\Delta t$ values, especially at $t_{1}=80 \mathrm{msec}$ and $t_{1}=100 \mathrm{msec}$. In these cases, performance seems to be completely determined by the $t_{2}$ value. The statistical analysis of the effects of $t_{1}$ and $t_{2}$ at $\Delta t<0\left(t_{2}=40 \mathrm{msec}, 60 \mathrm{msec}\right)$ confirmed this impression. The effect of $t_{2}$ was reliable $[F(1,3)$ $=40.6(\mathrm{p}<.011$, but, most interestingly, no effect of $\mathrm{t}_{1}\left(60,80,100 \mathrm{msec}\right.$ and $\mathrm{t}_{1}=\infty$, i.e., blank flash alone $)$ could be demonstrated $[F(3,9)=1.23$, n.s.].

\section{DISCUSSION}

Our results clearly demonstrate the recovery effect. Absolute performance and absolute amounit of recovery depend very strongly on $t_{1}$. However, the value of $t_{1}$ is of minor importance for the interval, $\Delta t$, at which recovery is found. This interval, $\Delta t$, was within 
the range of $0-40 \mathrm{msec}$. It is interesting to note the relatively wide range of $t_{1}$ values (from $t_{1}=10$ to $95 \mathrm{msec}$ ) at which other investigators found recovery (Dember \& Purcell, 1967; Dember et al., 1978; Kristofferson et al., 1979; Robinson, 1966; Schiller \& Greenfield, 1969). On the other hand, the range of $\Delta t$ values at which recovery was reported is smaller (10-55 msec). In spite of the large differences in experimental condition, these values agree well with our data.

Different results are obtained in the conditions in which $B$ precedes $M$. In such conditions, performance is completely determined by the interval between $T$ and $B\left(t_{2}\right)$.

Dember, Schwartz, and Kocak (1978) have proposed an explanation that is based on the existence of different temporal characteristics of the neuronal activity resulting from visual stimulation (Breitmeyer \& Ganz, 1976).

If "transient" activity resulting from the third flash, B, inhibits "sustained" activity of the second flash, $M$, before the sustained activity of $M$ and target integrate, pattern masking would be prevented. This theory offers a direct explanation for the major importance of the interval $\Delta t$ in our data. In addition, it predicts that optimal recovery will occur at a nonzero interval $\Delta t$. The asymmetrical influence of the order of presentation of $M$ and $B$ can also be explained in this theory. The transient activity generated by $\mathrm{B}$ simply inhibits the sustained activity resulting from target stimulation.

Yet, this approach leaves a number of questions unanswered. A first problem has been pointed out by Dember et al. (1978). Why should the inhibition of $M$ by $B$ at a short $t_{1}$ interval be so effective while no influence on target detectability could be found at the same $t_{2}$ value? Second, transient inhibition of targetsustained activity did not lead to a nonmonotonous masking curve. Why? A third problem is the mechanism by which the activation due to target and mask stimulations are kept separate for some time. Finally, it is not clear why there is no transient inhibition of the target by the mask.

Schiller and Greenfield (1969) have called attention to another mechanism that might be responsible for the recovery. They attribute the masking effect of a contour on another contour in close proximity to the indiscriminability of the responses to these contours generated by a restricted set of neurons. They reduced the similarity of a circular target and mask by selectively removing the contour of the mask. The response of cells sensitive to contours will be rather small when stimulated by a homogeneous field. As a consequence, the response pattern of contour-sensitive cells to a tight combination of the circular mask and ring will be very different from the response to the target alone.

Dember and Purcell (1967) and Kristofferson et al. (1979) have used masking conditions very similar to those of Schiller and Greenfield (1969). The results obtained by the former authors can therefore be well explained by the mechanisms described above.

Our results can be explained by these mechanisms as well. Both target and noise mask will give rise to strong responses in a set of contour-sensitive cells. A blank flash presented shortly after a patterned stimulus will reduce activity in contour-sensitive cells (Schiller, 1968). The shorter the interval between the patterned stimuli and the blank flash, the larger this reduction will be.

Recovery is the net effect of a large reduction of the activity in contour-sensitive cells generated by the mask and a smaller reduction of the activity generated by the target. In the absence of any direct influence of the third flash on the target, maximal and complete recovery will be found at the zero $\Delta t$ interval (Schiller \& Greenfield, 1969). At higher values of $t_{1}$ $\left(t_{1} \geqslant 80 \mathrm{msec}\right)$, this can be seen in our data. However, at lower values of $t_{1}\left(t_{1}<80 \mathrm{msec}\right)$, the optimal interval $\Delta t$ is nonzero. This might be due to the considerable increase of the influence of the blank flash on target detectability (see Figure 1B).

The asymmetrical influence of the order of presentation of the pattern mask and the blank flash on target detectability is easily understood. A blank flash following a target will reduce the activity in contoursensitive cells. On the other hand, a blank flash preceding a noise mask will also suppress mask-related activity in contour-sensitive cells. Accordingly, performance at short $t_{2}$ intervals will be determined almost completely by the contrast reduction resulting from the blank flash.

Our experiments can be considered as an extension of the experiments of Dember and his co-workers (1978). They reported large recovery effects which can completely be accounted for by the above explanation.

Even part of the negative results of Schurman and Eriksen (1969) may be explained by these arguments. They failed to find any recovery in a letter-identification task when they presented two blank masking flashes. One would not expect any recovery to occur because contour information due to the target is reduced twice. Indeed, they actually found a deteriorating effect of the second blank flash.

The apparently conflicting results of Robinson (1966, 1968) and the results obtained by Schurman and Eriksen (1969) may be reconciled by taking into account some of the findings of Schiller and Greenfield (1969). The disk sizes used by Robinson (1966) will have established contour interaction between target and first mask. On the other hand, the dimensions of his third flash are such that it will act mainly as a large blank flash (Schiller \& Greenfield, 1969). Later, Robinson (1968) used disks that were larger than in the 1966 experiments. Taking into account the results of Schiller and Greenfield (1969), we must con- 
clude that the masking effect of the first mask on the target was twofold: partly by contrast reduction and partly by contour interaction. The main influence of the third flash will no doubt have been contrast reduction. In the theory expounded above, we would not expect a large recovery, and, indeed, the recovery reported by Robinson (1968) seems to be somewhat smaller than in his 1966 experiments, especially at short target-mask intervals.

Schurman and Eriksen (1969) employed the same disk sizes as Robinson (1966), but with negative results. In order to understand their negative findings, it is useful to relate their masking results again to those of Schiller and Greenfield (1969). The results of both experiments fully agree with respect to the absence of any masking influence of the large disk (60 $\mathrm{min}$ ) on the detectability of the smaller target (15 min). However, Schurman and Eriksen (1969) report that their subjects could detect target presence perfectly when the target diameter was $15 \mathrm{~min}$ and mask diameter was $30 \mathrm{~min}$. This result contradicts the findings of Schiller and Greenfield (1969), who reported at least some masking influence in similar stimulus conditions (30-min mask), but using a simple yes/no detection task. The forced-choice procedure used by Schurman and Eriksen (1969) probably induced subjects to compare all possible cues as target presence. This includes differences in brightness and apparent movement, which allow perfect discrimination between the two alternatives. Next, Schurman and Eriksen (1969) reduced target luminance to introduce errors in the performance. This method does not favor the identification of target contours in particular. The introduction of a third, blank flash will give a net positive effect only when actual information about target contours is used.

Finally, considering a possible neurophysiological mechanism, we must keep in mind that the central notion here is the reduction of contour-related activity by a homogeneous flash. The very first steps in visual processing have this property. Both retina and lateral geniculate nucleus (LGN) show the laterally inhibitory structure that could give this effect (Robinson, 1966, 1968; Schiller \& Greenfield, 1969).

Schiller (1968) has demonstrated the effectiveness of a large flash in reducing the activity generated by a smaller patch in on-center LGN cells. He noted that the inhibitory structure is less pronounced in the retina than in the LGN. Besides, it is known that in conditions of low-level light adaptation, in particular the retinal inhibitory connections are weakened. All recovery experiments discussed here were run under such conditions.

Therefore, we conclude that the suggestion put forward by Schiller and Greenfield (1969) about the role of LGN cells appears to be very useful in explaining recovery induced by homogeneous flashes.

\section{REFERENCES}

Breitmeyer, B. G., \& Ganz, L. Implications of sustained and transient channels for theories of visual pattern masking, saccadic suppression, and information processing. Psychological Review, 1976, 83, 1-36.

Bryon, C., \& Banks, W. P. Patterned stimuli in disinhibition and backward masking. Bulletin of the Psychonomic Society, $1980,15,105-108$.

Dember, W. N., \& Purcell, D. G. Recovery of masked visual targets by inhibition of the masking stimulus. Science, 1967 . 157, 1335-1336.

Dember, W. N., Schwartz, M., \& Kocak, M. Substantial recovery of a masked visual target and its theoretical interpretation. Bulletin of the Psychonomic Society, 1978, 11, 285-287.

Kristofferson, A. B., Galloway, J., \& Hanson, R. G. Complete recovery of a masked visual target. Bulletin of the Psychonomic Society, 1979, 13, 5-6.

RoBisson, D. N. Disinhibition of visually masked stimuli. Science, 1966, 154, 157-158.

Rosinson, D. N. Visual disinhibition with binocular and interocular presentations. Journal of the Optical Society of America, 1968, 58, 254-257.

Schille R, P. H. Single unit analysis of backward visual masking and metacontrast in the cat lateral geniculate nucleus. Vision Research, 1968, 8, 855-866.

Schiller, P. H., \& Greenfield, A. Visual masking and the recovery phenomenon. Perception \& Psychophysics, 1969, 6, 182-184.

Schurman, D. L., \& Emiksen, C. W. Summation and interaction of successive masking stimuli in visual perception. American Journal of Psychology, 1969, 82, 320-322.

TuRvey, M. T. On peripheral and central processes in vision: Inferences from an information-processing analysis of masking with patterned stimuli. Psychological Review, 1973, 80, 1-52.

\section{NOTE}

1. After the preparation of this manuscript, a paper published by Bryon and Banks (1980) reported that recovery was induced by the presentation of consecutive patterned flashes. We consider these results to be a confirmation of Turvey's (1973) findings. In both experiments, the combination of Mask 1 and Mask 2 shows a relatively high contour density. Such stimulation is more like a homogeneous gray flash than like a highly confusable contoured pattern. When a contoured Mask 1 pattern is replaced by a homogeneous flash, recovery can be expected (Schiller \& Greenfield, 1969).

(Manuscript received January 26, 1981; accepted for publication May 14, 1981.) 